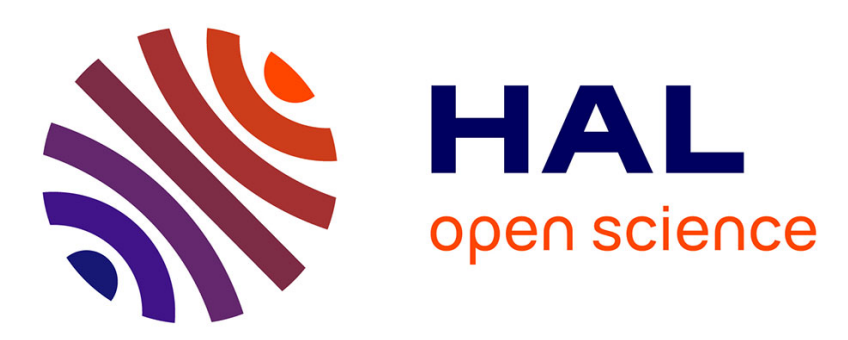

\title{
ÉLABORATION ET ÉVALUATION D'UNE LOI DE COMPORTEMENT BASÉE SUR L'ÉVOLUTION DE LA MICROSTRUCTURE
}

A. Rouxel, D. Bois

\section{- To cite this version:}

A. Rouxel, D. Bois. ÉLABORATION ET ÉVALUATION D'UNE LOI DE COMPORTEMENT BASÉE SUR L'ÉVOLUTION DE LA MICROSTRUCTURE. Journal de Physique IV Proceedings, 1991, 01 (C3), pp.C3-795-C3-802. 10.1051/jp4:19913112 . jpa-00249915

\section{HAL Id: jpa-00249915 https://hal.science/jpa-00249915}

Submitted on 1 Jan 1991

HAL is a multi-disciplinary open access archive for the deposit and dissemination of scientific research documents, whether they are published or not. The documents may come from teaching and research institutions in France or abroad, or from public or private research centers.
L'archive ouverte pluridisciplinaire HAL, est destinée au dépôt et à la diffusion de documents scientifiques de niveau recherche, publiés ou non, émanant des établissements d'enseignement et de recherche français ou étrangers, des laboratoires publics ou privés. 
Colloque C3, suppl. au Journal de Physique III, Vol. 1, octobre 1991

\title{
ÉLABORATION ET ÉVALUATION D'UNE LOI DE COMPORTEMENT BASÉE SUR L'ÉVOLUTION DE LA MICROSTRUCTURE
}

\author{
A. ROUXEL ${ }^{*}$ et D. BOIS** \\ *Ecole Nationale Supérieure de Mécanique, I, rue \\ de Ia Noë, 44072 Nantes, France \\ ** Aerospatiale-Division Engins Tactiques, \\ Châtillon-sous-Bagneux, France
}

Résumé: aexospatiale - Division Engins Tactiques a mené une étude dans le but d'améliorer la modélisation du comportement dynamique dans je domaine des faibles déformations plastíques (inférieures à 10\%). 'L'Ecole Nationale Supérieure de Mécanique de Nantes a réalisé les essais et proposé une modélisation prenant en compte l'évolution estimée de la microstructure. La première partie de l'étude consiste en la réalisation d'essais aux barres de Hopkinson en compression et l'élaboration du modèle pour deux matériaux: un acier 30NCD16 et un alliage de titane TA6V4. La seconde partie de l'étude réalisée à l'aerospatiale-Division Engins Tactiques, est l'implantation du modèle dans le code HEMP et son évaluation par des simulations numériques. Par comparaison avec les résultats expérimentaux, l'aptitude de cette loi à restituer le comportement dynamique des matériaux étudiés est discutée.

Abstract: A study has been carried by aerospatiale - Division Engins Tactiques to improve dynamic behaviour modelling in the range of small plastic deformation with strain value less than $10 \%$. Ecole Nationale Supérieure de Mécanique of Nantes has realized the dynamic mechanical testings and proposed a behaviour model based on estimated evolution of the microstructure. We describe first the tests performed with the split Hopkinson pressure bars and the development of the constitutive equations for 30 NCD16 steel and TA6V4 titanium alloy. In the second hand, these models have been introduced into the HEMP hydrocode, and numerical simulations are performed to test the constituve equations.

\section{INTRODUCTION:}

Lors du dimensionnement, par simulations numériques, d'un projectile devant résister à la perforation d'une cible mince en acier, on est confronté à la modélisation du comportement dynamique des matériaux constitutifs du projectile et de la cible. Si cette dernière subit des déformations importantes, il n'en est parfois pas de même de certaines régions du projectile, même si les vitesses de déformation dans ce dernier sont élevées. Il est donc nécessaire de disposer dans le code de calcul d'un modèle de comportement capable de restituer un domaine important de déformations et de vitesses de déformations.

Lors d'études antérieures $/ 1,2 /$, nous avions implanté dans le code de calcul HEMP, les lois de comportement de Johnson-Cook, de Lindholm et de Bodner-Partom /3-5/. Les 
modèles de comportement obtenus avec ces lois pour plusieurs aciers (35NCD16, 42CD4, ...) et un alliage de titane TA6V4 ont été validés par 1a comparaison des résultats d'essais d'impact de cylindres contre paroi rigide réalisés au CEA (Centre de Bruyères-Le-Châtel et CESTA) et la simulation numérique de ces essais. Nous avions constaté que si le comportement dynamique des matériaux étudiés était correctement simulé pour les grandes déformations, pour les faibles déformations (< $10 \%)$, $1 a$ variation de la contrainte en fonction de la vitesse de déformation est insuffisante. Bien que les phénomènes intervenant dans la microstruture soient importants pour la modélisation du comportement aux faibles déformations $/ 6 /$, un modèle empirique prendra difficilement en compte ces phénomènes. Des modèles basés sur la microstructure existent pour les monocristaux /7/. Aussi, une collaboration a été faite avec l'équipe de Plasticité Dynamique du Professeur CHIEM C.Y. à l'Ecole Nationale Supérieure de Mécanique de Nantes pour réaliser des essais, et élaborer un modèle de comportement basé sur l'évolution estimée de la microstructure, pour les polycristaux. Le but recherché étant la modélisation des déformations peu importantes: inférieures à $10 \%$.

\section{MATERIAUX ETUDIES:}

\begin{tabular}{|l|c|c|}
\hline Traitements Thermiques & Trempe/huile/850 & TA6V \\
\hline Revenu/air $/ 600^{\circ} \mathrm{C}$ & Trempe/eau $/ 850^{\circ} \mathrm{C}$ \\
\hline Revenu/air $/ 450^{\circ} \mathrm{C}$ \\
\hline Limite élastique & $1065 \mathrm{MPa}$ & $1065 \mathrm{MPa}$ \\
\hline Résistance à rupture & $1160 \mathrm{MPa}$ & $1170 \mathrm{MPa}$ \\
Allongement à rupture & $17.3 \%$ & $16.1 \%$ \\
\hline
\end{tabular}

\section{DISPOSITIFS EXPERIMENTAUX}

Les essais ont été réalisés sur les dispositifs aux barres de Hopkinson adaptés à la torsion et à la compression en dynamique, et sur une machine hydraulique de type MTS pour les essais en compression quasi-statique. Les méthodes classiques de dépouillement des essais aux barres de Hopkinson permettent, à partir des signaux fournis par des jauges extensométriques d'obtenir la courbe Contrainte / Déformation caractérisant l'échantillon.

Dispositif de barres de Hopkinson en compression dynamique: L'impact d'un projectile sur une barre d'incidence crée une onde élastique longitudinale qui se propage vers un échantillon de forme cylindrique placé entre la barre d'incidence et une barre de transmission (figure 1). Deux types d'essais peuvent être effectués:

- à vitesse de déformation constante (jusqu'à $3500 \mathrm{~s}^{-1}$ ) si le projectile, de section constante est constitué d'un seul matériau.

- à vitesse de déformation incrémentale ou décrémentale si le projectile est constitué de deux matériaux possédant des impédances différentes (par exemple: Marval 18 et Denal). Le profil des ondes obtenu dans ce cas est représenté figure 2 . 
Pour une vitesse d'impact de $13 \mathrm{~m} / \mathrm{s}$, on obtient des vitesses de déformation de 1 'ordre de $1000 \mathrm{~s}^{-1}$ et $2000 \mathrm{~s}^{-1}$ pour un échantillon de $4.3 \mathrm{~mm}$ de long.

Le dispositif de barres de Hopkinson peut également être utilisé pour mesurer le

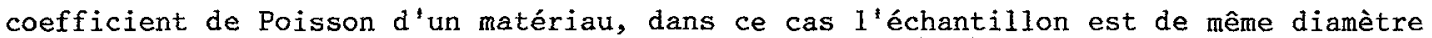
que les barres $(20 \mathrm{~mm})$. Sur l'éprouvette des jauges de déformation sont collées, parallèlement et perpendiculairement à l'axe de sollicitation. Le coefficient de Poisson est le rapport entre les déformation élastiques transversale et longitudinale. Nous avons obtenu un coefficient de Poisson expérimental de 0.38 pour le $30 \mathrm{NCD} 16$ et de 0.32 pour le TA6V, sur un ensemble de 12 mesures.

Essai en compression quasi-statique: Une machine de compression conventionnelle, de type MTS est utilisée pour les essais en compression à des vitesses de déformation comprises entre $10^{-3}$ et $10^{1} \mathrm{~s}^{-1}$.

Remarques: $\mathrm{Ni}$ le module de Young, ni la limite d'élasticité de type conventionnelle peuvent être mesurés au cours des essais sur barres de Hopkinson. La partition des déformations totales en composantes élastique et plastique n'est alors possible qu'au prix d'hypothèses sur la valeur du module de Young (conservation de la valeur statique). Dans le cas de matériaux métalliques il est raisonnable de supposer le module d'élasticité pratiquement insensible à la vitesse de déformation.

Après avoir mesuré le module d'élasticité expérimental, les résultats sont présentés sous forme de courbes Contrainte/Déformation plastique vraies, c'est à dire en considérant que 1 'échantillon se déforme à volume constant.

\section{MODELISATION DU COMPORTEMENT DU 30NCD16}

Les résultats expérimentaux des essais en compression statique et dynamique dans la gamme des vitesses de déformation de $10^{-3}$ à $3.10^{3} \mathrm{~s}^{-1}$ montrent qu'il existe trois zones distinctes sur les courbes Contrainte/Log (Vitesse de Déformation) à déformation constante, ces zones correspondent aux micromécanismes de déformation athermique, thermiquement activé et de frottement visqueux (respectivement régions I, II, III sur la figure 3).

L'équation proposée $f\left(\sigma, \varepsilon_{p}, \dot{\varepsilon}, s\right)$ relie entre elles des grandeurs macroscopiques mesurées durant les essais mécaniques à des grandeurs microscopiques supposées du comportement des dislocations. (s définit les paramètres microstructuraux).

La contrainte athermique: Les résultats expérimentaux en compression quasistatique indiquent que pour $\dot{\varepsilon}<10^{-1} \mathbf{s}^{-1}$, le comportement du 30NCD16 est défini par le processus athermique. La contrainte n'évolue pas en fonction de la vitesse de déformation. Ces résultats expérimentaux vont nous permettre de déterminer la relation $\sigma_{\mu}$ en fonction de la déformation plastique.

L'écrouissage du matériau est directement lié à l'évolution de la microstructure par sa densité de dislocations. La multiplication des dislocations accrô̂t le champ des contraintes à grande distance.

L'effet de l'écrouissage est décrit par la relation: $\sigma_{\mu}=\mathbb{E}(\mathrm{T})$ b $\rho^{1 / 2}$

$E(T)$ est le module de Young $(E(293 \mathrm{~K})=204048 \mathrm{MPa})$, b: le vecteur de Burgers

(b $\left.=2.48 \quad 10^{-8} \mathrm{~cm}\right)$.

La densité de dislocations évolue avec la déformation plastique. Cette évolution peut s'écrire: $\rho=\rho_{\mathrm{i}}+\mathrm{Cm} / \mathrm{Cr}\left[1-\exp \left(-\operatorname{Cr} \varepsilon_{\mathrm{p}}\right)\right]$ 
$\rho_{i}$ est la densité initiale de dislocations. Cm et $\mathrm{C} r$ représentent respectivement les coefficients multiplicatifs et de réduction des dislocations dus à la déformation plastique accumulée. Les valeurs numériques déduites des résultats expérimentaux sont, pour le 30NCD16: $\rho_{\mathrm{i}}=3.8010^{10} / \mathrm{cm}^{2}, \mathrm{Cm}=4.810^{11} / \mathrm{cm}^{2}, \mathrm{Cr}=22$.

La figure 4 compare l'évolution de la contrainte athermique par rapport à la déformation plastique vraie, obtenue expérimentalement en compression quasi-statique, au modèle proposé pour les vitesses de déformation inférieures à $10^{-1} \mathrm{~s}^{-1}$.

- La contrainte thermiquement activée: Pour les vitesses de déformation supérieures à $10^{-1} \mathrm{~s}^{-1}$, la contrainte est une fonction du logarithme de la vitesse de déformation. La contrainte effective $\sigma^{*}$ est la différence entre la contrainte appliquée $\sigma$ et la contrainte athermique $\sigma_{\mu}: \sigma^{*}=\sigma-\sigma_{\mu}$

La contrainte effective est la contrainte qui aide les dislocations à franchir les barrières au moyen des fluctuations thermiques.

L'analyse effectuée par différents auteurs /8/ indique que les dislocations dans les structures CC se déplacent par la formation thermiquement activée de paires de décrochements sur les potentiels de Peierls. En nous basant sur le modèle de Peierls, nous avons écrit notre modèle dans le domaine thermiquement activé avec l'équation:

$$
\Delta \mathrm{G}_{\mathrm{p}}=2 \mathrm{H}_{\mathrm{k}}(\mathrm{T})\left(1-\left(\sigma^{*} / \sigma_{\mathrm{p}}^{*}\right) \mathrm{p}\right)
$$

avec $p=1, \Delta G p$ est l'énergie libre d'activation pour former un double décrochement et vaincre les potentiels de Peierls $/ 9,10 /, 2 \mathrm{H}_{\mathrm{k}}(\mathrm{T})$, l'énergie du double décrochement $\left(2 \mathrm{H}_{\mathrm{k}}(293 \mathrm{~K})=0,559 \mathrm{eV}\right)$ et $\sigma_{\mathrm{p}}^{*}(\mathrm{~T})$, la contrainte de Peierls $\left(\sigma_{\mathrm{p}}^{*}(293 \mathrm{~K})=675 \mathrm{MPa}\right)$ dépendent de la température par le module d'élasticité $\mathrm{E}(\mathrm{T})$. Soit:

$2 \mathrm{H}_{\mathrm{k}}(\mathrm{T})=2 \mathrm{H}^{\circ}{ }_{\mathrm{k}} \mathrm{E}(\mathrm{T}) / \mathrm{E}^{\circ}$ et $\sigma_{\mathrm{p}}^{*}(\mathrm{~T})=\sigma_{\mathrm{p}}^{\circ} \mathrm{E}(\mathrm{T}) / \mathrm{E}^{\circ}, 1^{\prime}$ exposant $1^{\circ}$ indique une température égale à $0 \mathrm{~K}$.

A zéro degré, 1 'énergie thermique est nulle, toute 1 'énergie nécessaire pour vaincre une dislocation doit être fournie mécaniquement, dans ce cas $\Delta \mathrm{G}_{\mathrm{p}}=2 \mathrm{H}_{\mathrm{k}}^{\circ}$ et $\sigma^{\star}=\sigma_{\mathrm{p}}^{\circ}$.

Lorsque $\sigma^{*}$ est disponible, $l^{\prime}$ énergie thermique varie entre $0<\Delta \mathrm{Gp}<2 \mathrm{H}_{k}^{\circ}$, pour $\sigma_{p}^{\circ}>\sigma^{*}>0$.

Pour une température suffisamment élevée, $\mathrm{T}>$ Tcritique, l'énergie thermique $\mathrm{n}$ 'est plus nécessaire pour vaincre un obstacle, $\Delta \mathrm{Gp}=0$. La modélisation du comportement thermiquement activé n'est applicable qu'aux températures inférieures à la température critique $T_{c}$ et pour des vitesses de déformation supérieures à $\dot{\varepsilon}_{c}$, avec $\dot{\varepsilon}_{c}\left(T_{c}\right)$. La contrainte thermique s'écrit donc: $\sigma^{*}=\sigma_{\mathrm{p}}^{*}\left[1-\left(\mathrm{kT} / 2 \mathrm{H}_{\mathrm{k}} \ln \left(v_{\mathrm{p}} / \dot{\varepsilon}\right)\right]\right.$ ou $v_{p}=\rho b^{2} v_{D}$ avec $v_{D}=10^{13} \mathrm{~s}^{-1}$, fréquence de Debye. $k=8.61410^{-5} \mathrm{eV} / \mathrm{K}$ est $1 \mathrm{a}$ constante de Boltzmann.

La température critique est calculée par la relation: Tcrit $=2 \mathrm{H}_{\mathrm{k}} / \mathrm{k} / \operatorname{Ln}\left(\nu_{\mathrm{D}} / \dot{\varepsilon}\right)$ Pour $\mathrm{T}<$ Tcrit: $\sigma=\sigma_{\mu}+\sigma^{*}$, sinon $\sigma=\sigma_{\mu}$

- La contrainte visqueuse: Pour les vitesses de déformation supérieures à $10^{3} \mathrm{~s}^{-1}$ en compression, la contrainte est directement proportionnelle à la vitesse de déformation: $\sigma_{v}=\sigma-\sigma_{\mu}-\sigma^{*}=\alpha \dot{\varepsilon}$, avec $\alpha=B_{v} / b^{2} / \rho$

Les résultats expérimentaux indiquent que $B_{v}$, le coefficient de viscosité moyen est de l'ordre de $2.54310^{-6} \mathrm{MPa} . \mathrm{s}$.

La figure 3 compare le modèle de comportement proposé pour le 30NCD16 aux résultats obtenus en compression dans la gamme des vitesses de déformation de $10^{-4}$ à $3.10^{3} \mathrm{~s}^{-1}$ à $2.5 \%$ de déformation plastique. 
Le modèle s'adapte correctement aux résultats expérimentaux pour les déformations inférieures à $16 \%$, dans la gamme des vitesses de déformation étudiées.

\section{MODELISATION DU COMPORTEMENT DU TA6V}

Les résultats expérimentaux des essais sur le TA6V en compression statique et dynamique ont servi de base à la réalisation de ce modèle. Ces résultats montrent que même aux plus faibles vitesses de déformation, de $1^{\prime}$ ordre de $10^{-1} \mathrm{~s}^{-1}$, le TA6V se déforme déjà suivant le micromécanisme de déformation thermiquement activé.

Nos résultats expérimentaux sont en accord sur l'évolution de la contrainte en fonction de la vitesse de déformation avec les résultats de Harding (1981), Meyer et Chiem (1984).

Harding $/ 11 /$ indique que la température critique est de $l^{\prime}$ ordre de $500^{\circ} \mathrm{C}$, c'est à dire qu'au-dessus de cette température, la contrainte athermique $\sigma_{\mu}$ intervient seule dans les mécanismes de déformation et ne peut être définie clairement que pour des températures d'essais supérieures à $500^{\circ} \mathrm{C}$. Les essais en température ne faisant pas partie de ce travail nous avons établi le modèle en tenant compte des remarques de Harding.

Tanaka et al./13/ font remarquer que dans le cas des alliages de titane, les composantes thermique et athermique de la contrainte sont du même ordre de grandeur. L'énergie d'activation $\Delta G$ est définie par:

$$
\Delta \mathrm{G}=\mathrm{D}\left[1-\ln \left(\left(\sigma^{*} / \sigma_{\mathrm{p}}\right)\right]=\mathrm{kT} \ln \left(v_{\mathrm{p}} / \dot{\varepsilon}\right)\right.
$$

Ces relations indiquent que la contrainte effective peut être écrite par:

$$
\sigma^{*}=\sigma_{\mu} \exp (1)\left(\dot{\varepsilon} / v_{\mathrm{p}}\right)^{\mathrm{kT} / \mathrm{D}}
$$

avec $\mathrm{b}=2.9110^{-8} \mathrm{~cm}$ le vecteur de Burgers du titane, 1'équation de la contrainte athermique est: $\sigma_{\mu}=\mathrm{E}$ b $\rho^{1 / 2}$ avec $\rho=\rho_{\mathrm{i}}+\operatorname{Cm} / \operatorname{Cr}\left(1-\exp \left(-\operatorname{Cr} \varepsilon_{\mathrm{p}}\right)\right)$

Les valeurs numériques obtenues sont: $\rho_{\mathrm{i}}=2.9910^{10} / \mathrm{cm}^{2}:$ la densité initiale de dislocations, $\mathrm{Cm}=1.2910^{11} / \mathrm{cm}^{2}$ : le coefficient multiplicatif des dislocations, $\mathrm{Cr}=34.5:$ le coefficient de diminution des dislocation, $\mathrm{E}(\mathrm{Tamb})=114058 \mathrm{MPa}$.

L'expression du terme pré-exponentiel: $v_{p}$ du TA6V est identique à celle du 30NCD16. Pour les vitesses de déformation comprises entre $10^{-3} \mathrm{~s}^{-1}$ et $10 \mathrm{~s}^{-1}$, une bonne approximation des contraintes en fonction de la vitesse de déformation est obtenue par une équation du type: $\sigma=A \dot{\varepsilon}^{m}$. La sensibilité à la vitesse de déformation s'exprime en fonction de la déformation par: $\mathrm{m}\left(\varepsilon_{\mathrm{p}}\right)=0.016-0.0433 \varepsilon_{\mathrm{p}}$, en identifiant $\mathrm{m}\left(\varepsilon_{\mathrm{p}}\right)$ à $\mathrm{kT} / \mathrm{D}$, nous avons déterminé $\sigma^{*}$.

De même que pour le 30NCD16, pour les vitesses de déformations supérieures à $10^{3} \mathrm{~s}^{-1}$, le comportement du TA6V est caractérisé par le micromécanisme de frottement visqueux. $\sigma_{v}=\alpha \dot{\varepsilon}$ avec: $\alpha=B_{v} / b^{2} / \rho$ expérimentalement: $B_{v}=4.2310^{-6} \mathrm{MPa} . \mathrm{s}$.

La figure 5 compare la modélisation du comportement du TA6V aux résultats expérimentaux en compression dans la gamme des vitesses de déformation de $10^{-3}$ à $3.10^{3} \mathrm{~s}^{-1}$ à $5 \%$ de déformation.

Le modèle ne convient que pour les déformations inférieures à $16 \%$. Pour valider complètement ce modèle il conviendrait de faire des essais à haute température pour connaître exactement la contrainte athermique. 


\section{SIMULATION DES ESSAIS}

Nous avons simulé certains essais en compression dynamique aux barres de Hopkinson, à vitesse de déformation constante pour l'acier 30NCD16 et l'alliage de titane TA6V. Le modèle utilisé, à une seule maille, est uniaxial en contrainte et multiaxial en déformation. La compression de l'échantillon s'effectue à volume constant en imposant une vitesse aux noeuds de la maille. Cette vitesse évolue en fonction du temps et est choisie de façon à obtenir une vitesse de déformation constante pendant la durée de la simulation. Les résultats des simulations numériques sont comparés à ceux de 1 'analyse rationnelle des essais.

Les figures $6,7,8$ donnent une comparaison des courbes Contrainte/Déformation plastique expérimentales et numériques pour le 30 NCD16 aux vitesses de déformation: $998 \mathrm{~s}^{-1}, 1790 \mathrm{~s}^{-1}$ et $3145 \mathrm{~s}^{-1}$.

Les comparaisons simulations/essais pour le TA6V sont données sur les figures 9,10 et 11 pour $1337 \mathrm{~s}^{-1}, 2066 \mathrm{~s}^{-1}$ et $2876 \mathrm{~s}^{-1}$.

Les écarts entre simulations et résultats expérimentaux sont faibles, tant pour le TA6V que pour le 30NCD16, et ce pour un taux de déformation pouvant dépasser 10\%. Au voisinage de la limite élastique, les écarts sont légèrement plus importants pour le TA6V que pour le 30NCD16.

\section{CONCLUSION}

La modélisation décrite précédemment apporte une amélioration pour la simulation du comportement dynamique du $30 \mathrm{NCD} 16$ et du TA6V, pour des déformations inférieures ou égales à $10 \%$ par rapport aux modèles semi-empiriques.

L'implantation de ces modèles dans un code de calcul est relativement aisée. Toutefois, l'utilisation fréquente de ces modèles et leur emploi pour d'autres matériaux nécessite des outils d'aide à la détermination automatique des coefficients.

\section{REMERCIEMENTS}

Les auteurs tiennent à remercier Monsieur le Professeur CHIEM de l'ENSM pour ses conseils et la direction de ces travaux. Nos remerciements s'adressent aussi à I'aerospatiale pour avoir financé cette étude et à Monsieur GRAVE pour ses conseils.

\section{REFERENCES}

1- D. BOIS, A. GRAVE: DYMAT 85 Paris, J. de Phys C5, n8, T.46 (1985)

2- D. BOIS, A. GRAVE, M.L. MEYNIEL: Journées Détonique 86, Bourges (1986)

3- G.R. JOHNSON, W.H. COOK: $7^{\text {th }}$ Int. Symp. on ballistics (1983)

4- U.S. LINDHOLM: J. Mech. Phys. Solids, vol.12 (1964)

5- S.R. BODNER, Y. PARTOM: J. of Eng. Mat. and Tech., Trans. of ASME, Vol.101 (1979)

6- C.Y.CHIEM: EXPLOMET 90, San Diego (1990)

7- J.P. DUBOIS, P. BLINOT, C.Y. CHIEM: DYMAT 85 Paris, J. Phys. C5, n8, T.46 (1985)

8- H. CONRAD: J. of Metals, July (1964)

9- M. CAGNON: Thèse de Docteur Es Sciences Physiques, Univ. Paris-Sud (1973)

10- O. VOHRINGER: Ecole d'été, ENSM, Nantes (1989)

11- J. HARDING: Ecole d'été, ENSM, Nantes (1989)

12- L.W. MEYER, C.Y. CHIEM: 5th Conf. on Titanium, Munich (1984)

13- K. TANAKA, K. OGAWA, T. NOJIMA: IUTAM Symposium, Tokyo (1977) 


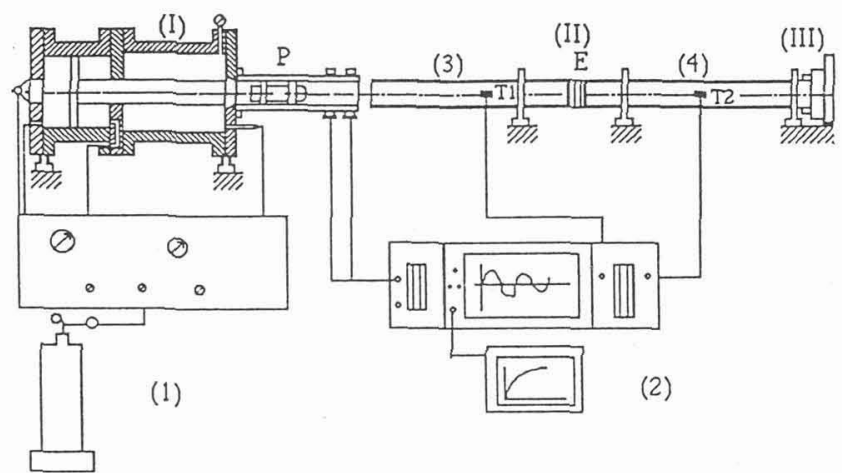

(I) : Lanceur pneumatique

(1) : Pupitre de commande et bouteille daazote

(2) : Chaînes électronique et informatique (ii) : Barres de Hopkinson

(3) : Barre d'incidence

(4) : Barre de transmission
(III) : Absorbeur de choc T1,T2 : Stations de jauges

P : Projectile

Figure 1: Schéma du dispositif expérimental des barres de Hopkinson en compression

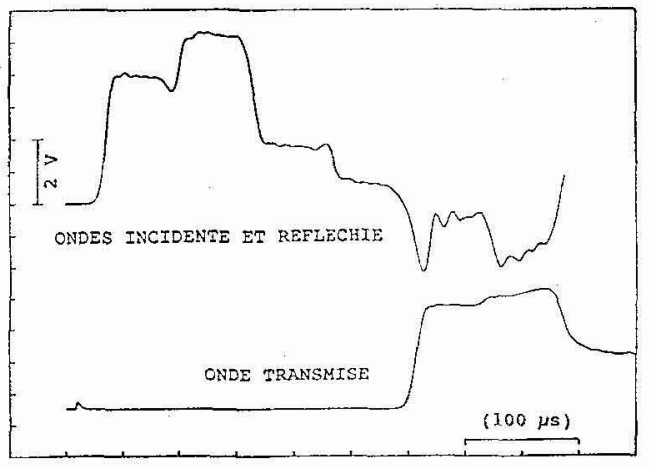

Figure 2: Profil des ondes enregistrées lors d'un essai en compression avec un projectile double impédance.

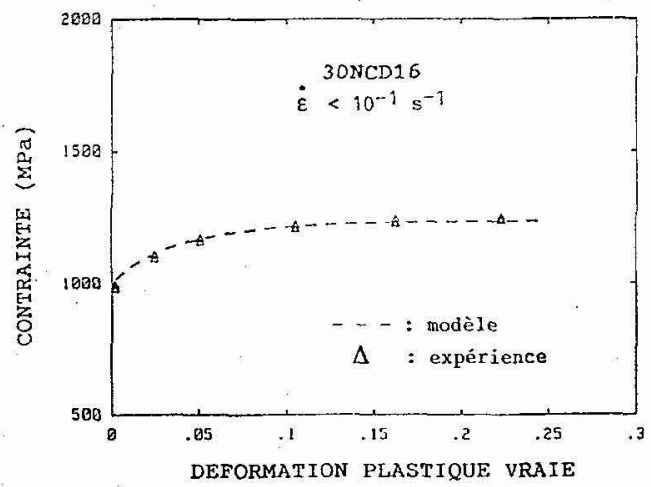

Figure 4: Contrainte/Déformation du 30NCD16 pour $\dot{\varepsilon}<10^{-1} \mathrm{~s}^{-1}$.

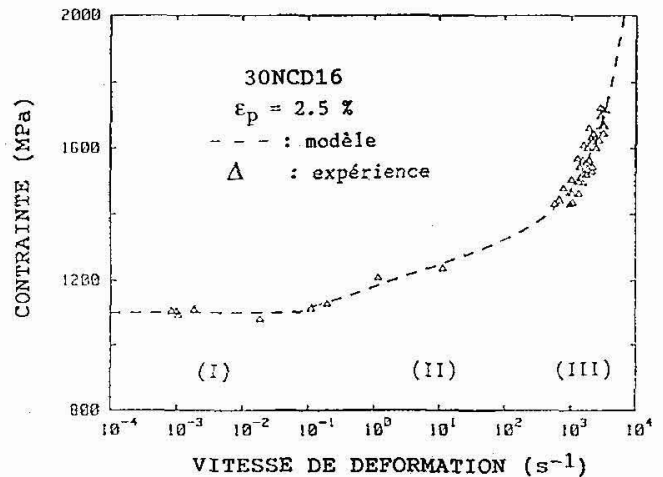

Figure 3: Contrainte/Log(Vitesse de déformation) du $30 \mathrm{NCD} 16$ à $2.5 \%$ de déformation plastique.

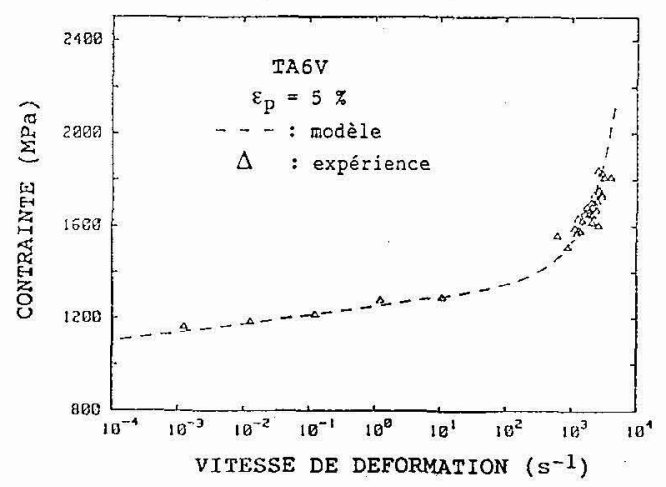

Figure 5: Contrainte/Log(Vitesse de déformation) du TA6V à $5 \%$ de déformation plastique. 


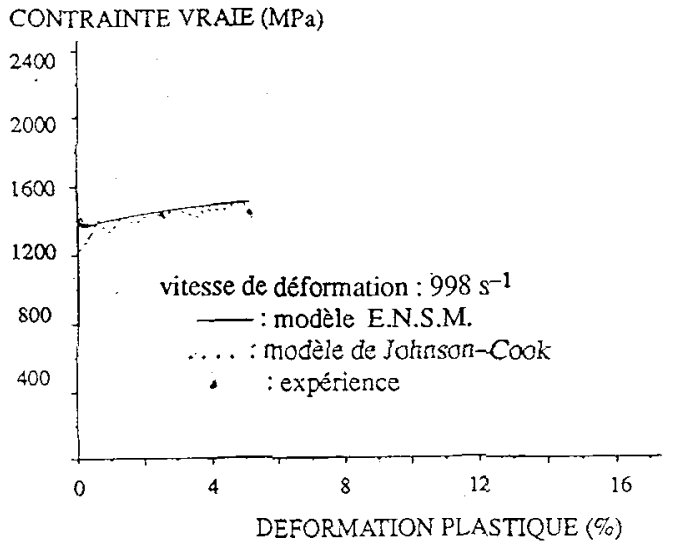

fig. 6: comparaison simulation/expérience pour le 30NCD16 avec les modeles de Johnson-Cook et de l'E.N.S.M.

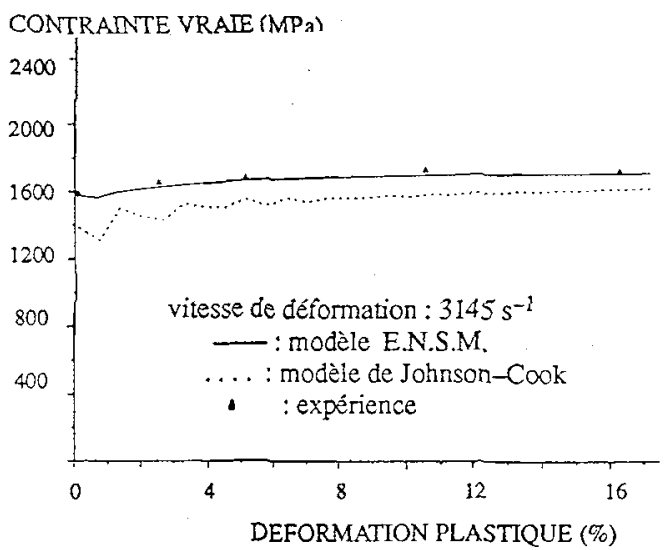

fig. 8 : comparaison simulation/expérience pour le 30NCD16 avec les modèles de Johnson-Cook et de l'E.N.S.M.

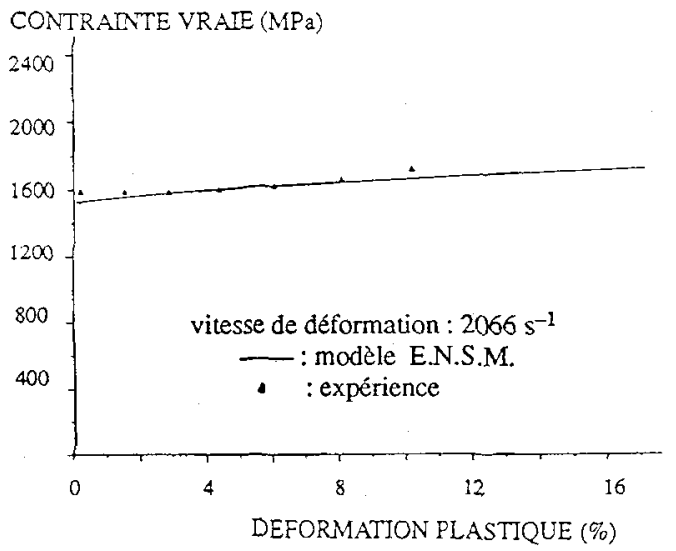

fig. 10: comparaison simulation/expérience pour le TA6V4 avec le modèle de l'E.N.S.M.

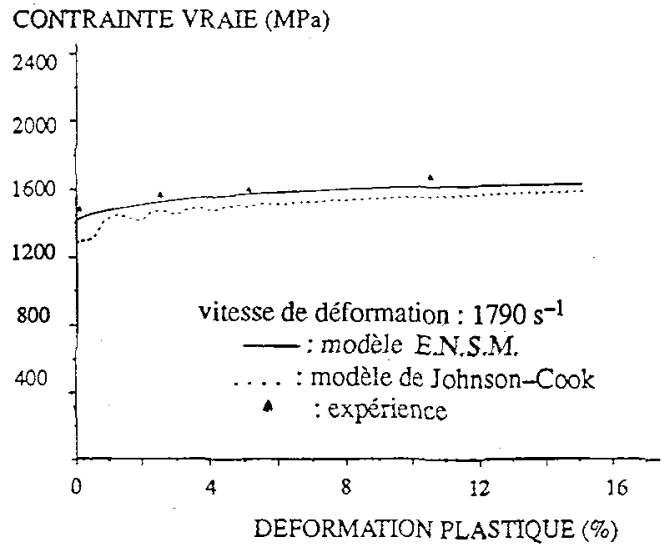

fig. 7 : comparaison simulation/expérience pour le 30 NCD16 avec les modèles de Johnson-Cook et de l'E.N.S.M.

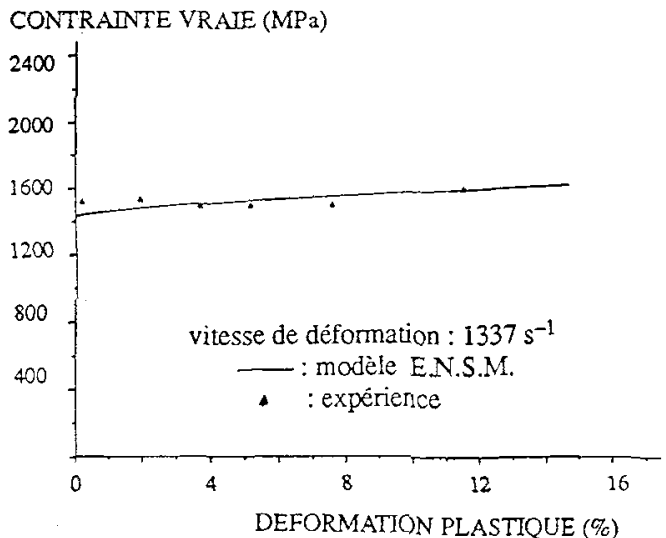

fig. 9 : comparaison simulation/experience pour le TA6V4 avec le modèle de l'E.N.S.M.

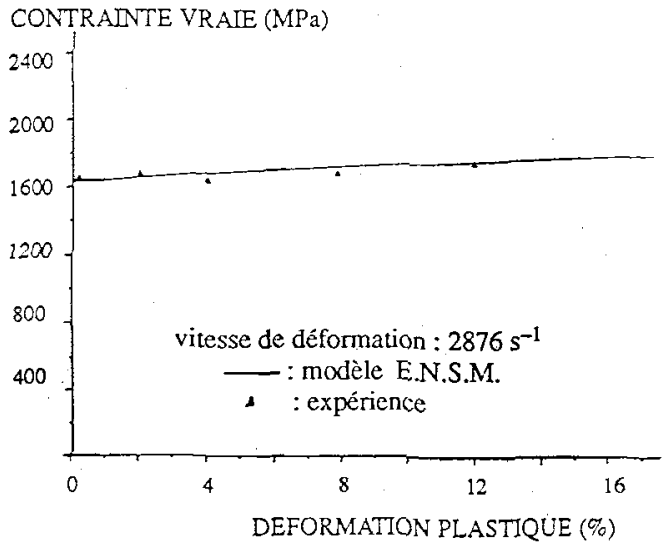

fig. 11 : comparaison simulation/expérience pour le TA6V4 avec le modèle de l'E.N.S.M. 